\title{
Kann Europa gelingen? Vorhaben und Chancen der deutschen Ratspräsidentschaft
}

\author{
Daniel Göler und Mathias Jopp*
}

Deutschland hat die EU-Ratspräsidentschaft in einem schwierigen Umfeld übernommen. Seit den ablehnenden Referenden zum Verfassungsvertrag in Frankreich und den Niederlanden steckt die Europäische Union in einer Krise, aus der sich auch nach eineinhalb Jahren kein richtiger Ausweg abzeichnet. ${ }^{1}$ Kernproblem ist hierbei die ungelöste Frage einer grundlegenden EU-Reform, deren Dringlichkeit sich mit dem am ersten Januar 2007 erfolgten Beitritt Rumäniens und Bulgariens weiter verschärft hat. Allerdings haben die Referenden auch gezeigt, dass Europa auf nicht unerhebliche Akzeptanzprobleme in der Bevölkerung stößt, die sich auf Dauer als problematisch für den Integrationsprozess erweisen könnten. Entsprechend dem von der Europäischen Kommission zur Überwindung der Akzeptanzkrise propagierten „double-track-approach“ gilt es nunmehr, neben Versuchen zur Lösung der Verfassungsfrage konkrete und bürgernahe Politikvorhaben in die Tat umzusetzen (Ansatz des „Europe des projets“2). Immer wieder genannt werden hierbei zum Beispiel die Bereiche europäische Energiepolitik, Asyl- und Einwanderungspolitik oder die weitere Ausgestaltung der Lissabonstrategie. Nimmt man hinzu, dass auch in der Außen- und Sicherheitspolitik und im Bereich der EU-Nachbarschaftspolitik große Herausforderungen anstehen, ergibt sich für die deutsche Ratspräsidentschaft ein erheblicher Handlungsbedarf.

Verkompliziert wird diese Ausgangslage durch eine Phase der Führungsschwäche in Europa. Frankreich befindet sich im Wahlkampf, von Großbritannien sind vor der Amtsübergabe Tony Blairs auf seinen Nachfolger keine Impulse zu erwarten und in den Niederlanden haben die letzten Wahlen ein Patt zwischen den politischen Lagern ergeben. Die polnische Regierung verfolgt europapolitisch einen Kurs, den man bestenfalls als eigenwillig bezeichnen kann; in Ungarn dauert die Regierungskrise an und die slowakische Regierungskoalition hat sich durch die Einbeziehung der ultrarechten SNS (Slowakische Nationalpartei) in Misskredit gebracht. In dieser Situation sind die Anforderungen und Erwartungen an die deutsche Ratspräsidentschaft besonders hoch. Pointierter könnte man auch sagen, dass die deutsche Bundesregierung zurzeit die einzig wirkliche Kraft ist, die es schaffen kann, dass Europa gemeinsam gelingt. ${ }^{3}$

Das ähnlich lautende an den Gemeinschaftssinn appellierende Programm der deutschen Ratspräsidentschaft, das an die Arbeiten der vorangegangenen Präsidentschaften und die Jahresplanung der Kommission anknüpft, beinhaltet weiterreichende Zielsetzungen unter anderem in der „Gestaltung der wirtschaftlichen, sozialen und ökologischen Zukunft Euro-

* Dr. Daniel Göler, wissenschaftlicher Mitarbeiter, Institut für Europäische Politik, Berlin; Lehrbeauftragter an der Freien Universität Berlin.

Dr. Mathias Jopp, Direktor des Instituts für Europäische Politik, Berlin; Lehrbeauftragter an der Universität Tübingen und der Europauniversität Viadrina, Frankfurt/Oder.

1 Vgl. Daniela Schwarzer: Lehren aus den gescheiterten Verfassungsreferenden, SWP-Aktuell 2005/A 23.

2 Vgl. José Manuel Barroso: Speech to the European Parliament prior to the European Council of 15 and 16 June 2006, S. 2, abrufbar unter: http://ec.europa.eu/commission_barroso/president/pdf/speech_20060614_en.pdf (letzter Zugriff: 17.12.2006).

3 Vgl. Bundesregierung: Europa gelingt gemeinsam. Präsidentschaftsprogramm 1. Januar - 30. Juni 2007, abrufbar unter: www.eu2007.de (letzter Zugriff: 26.01.2007). 
pas“, des „Raums der Freiheit, der Sicherheit und des Rechts“ sowie der „Gestaltung der gemeinsamen Außen- und Sicherheitspolitik, der Außenwirtschaftspolitik und der Entwicklungspolitik“.4

Freilich sollten die Erwartungen an die deutsche Präsidentschaft nicht überdehnt werden. Die Rolle einer Ratspräsidentschaft konzentriert sich in erster Linie auf das Vorsitz- und Agendamanagement, die Mediation und Konsensbildung zwischen den Mitgliedstaaten sowie interne und externe Repräsentations- und Sprecher-Aufgaben. ${ }^{5}$ Darüber hinaus kann die deutsche Präsidentschaft, wenn die Umstände günstig sind und ihre Planungen nicht durch eine massive internationale Krise konterkariert werden, in einigen Bereichen neue Initiativen ergreifen oder wichtige Projekte anschieben. Da der Zeitraum von nur sechs Monaten sehr kurz ist, hat die Bundesregierung sich mit der folgenden portugiesischen und slowenischen Präsidentschaft eng abgestimmt und mit ihnen ein gemeinsames 18-Monate Programm entworfen. ${ }^{6}$ Selbst wenn es also in etlichen Bereichen nicht gelingen wird, fertige Lösungen zu erarbeiten, so können doch Richtungsentscheidungen getroffen und Grundlagen für die Arbeiten der folgenden Präsidentschaften gelegt werden. ${ }^{7}$ Begünstigend wird sich für die Bundesregierung zudem auswirken, dass sie - wie schon bei ihrer letzten EURatspräsidentschaft im Jahre 1999 - zugleich den Vorsitz der G8 inne hat. Vor allem in der Handels- und Entwicklungspolitik, dem Umwelt- und Klimaschutz, der Energiesicherheit sowie in zentralen Fragen des internationalen Krisenmanagements bieten sich besondere Chancen für Synergien und Zusammenarbeit zwischen der EU und den nicht der EU angehörenden G8-Staaten (Kanada, USA, Japan und Russland).

Betrachtet man neben dem eigentlichen Präsidentschaftsprogramm der Bundesregierung ${ }^{8}$ das Legislativ- und Arbeitsprogramm der Kommission für 2007, ${ }^{9}$ so lassen sich allein in Letzterem 38 wichtige Legislativ-Vorhaben erkennen, von denen 16 während der deutschen Ratspräsidentschaft voran gebracht oder abgeschlossen werden sollen. ${ }^{10}$ Gerade hier liegt ein Hauptteil der Arbeit einer Präsidentschaft, dass heißt in der Koordinierung der täglichen Arbeit in den einzelnen Ratsformationen und der ,normalen' Rechtsetzung. Hinzu kommen noch circa 153 Fachkonferenzen, Beamten- und Expertentreffen der Ratspräsidentschaft (zu Themen wie Gesundheit, Familie, Immigrantenintegration, europäisches Sozialmodell, Energie, Umwelt), ${ }^{11}$ komplexe Gipfeltreffen und Verhandlungen insbesondere mit Russland und den USA sowie drei Treffen des Europäischen Rates unter Einschluss der Feierlichkeiten zum 50-jährigen Bestehen der Römischen Verträge. Letztgenanntes Treffen bietet die Chance, durch eine Berliner Erklärung den Sinn und den Nutzen des europäischen Integrationsprozesses in den letzten fünf Dekaden für die Bürger deutlich herauszustellen und vor al-

4 Vgl. die entsprechende Gliederung des deutschen Präsidentschaftsprogramms: Bundesregierung: Europa gelingt gemeinsam.

5 Zu den Aufgaben von Ratspräsidentschaften vgl.: Adriaan Schout/Sophie Vanhoonacker: Evaluating Presidencies of the Council of the EU. Revisiting Nice, in: Journal of Common Market Studies 5/2006, S. 1053-1056.

6 Rat der Europäischen Union: Achtzehnmonatsprogramm des deutschen, des portugiesischen und des slowenischen Vorsitzes, 11.12.2006, abrufbar unter: http://www.eu2007.de/includes/Download_Dokumente/Trio-Programm/triodeutsch.pdf (letzter Zugriff: 26.01.2007).

7 So Staatssekretär Joachim Wuermeling auf der Konferenz „Energy security in an uncertain world“, 05.10.2006 in Berlin.

8 Vgl. Bundesregierung: Europa gelingt gemeinsam.

9 Vgl. Europäische Kommission: Legislativ- und Arbeitsprogramm der Kommission für 2007, KOM(2006)629 endgültig.

10 Zudem sollen zahlreiche außerhalb des Legislativ- und Arbeitsprogramms der Kommission für 2007 stehenden Legislativ-Vorhaben im Semester der deutschen Ratspräsidentschaft abgeschlossen werden, siehe dazu: http:// ec.europa.eu/atwork/programmes/docs/forward_programming.pdf (letzter Zugriff: 26.01.2007).

11 Die Terminübersicht ist abrufbar unter: http://www.eu2007.de/de/Meetings_Calendar/index.html (letzter Zugriff: 26.01.2007). 
lem auch Perspektiven für die Zukunft Europas aufzuweisen. Angesichts der Fülle der auf der Agenda stehenden Punkte wird für den Erfolg der deutschen Präsidentschaft entscheidend sein, inwiefern es ihr gelingt, eine gesunde Mischung aus effizienter Bewältigung der Alltagspolitik und ,Lösungsansätzen für die großen Fragen` zu erzielen.

\section{Europäische Wirtschafts- und Sozialpolitik}

Zwei Bereiche der EU-Politik, die immer wieder in der Kritik stehen, sind das europäische Sozialmodell und die Verbesserung der Wirtschaftsentwicklung in Europa. Zentraler Ansatz der EU ist in beiden Bereichen die Lissabon-Strategie, die jedoch aufgrund ihrer aus heutiger Sicht unrealistischen Zielsetzung, Europa bis zum Jahr 2010 zum wettbewerbsfähigsten wissensbasierten Wirtschaftsraum zu entwickeln und somit die Beschäftigungsprobleme zu lösen, häufig als Ausdruck fehlgeleiteter europäischer Politik wahrgenommen wird. ${ }^{12}$ Nicht zuletzt wegen solcher Kritik wurde die Lissabon-Strategie 2005 einer grundlegenden Revision unterzogen, die trotz der fortbestehenden Schwächen der (offenen) Regierungskoordination darauf abzielt, die einzelnen Maßnahmen und Programme verbindlicher zu gestalten. In diesem Rahmen verpflichteten sich die Mitgliedstaaten, nationale Reformprogramme (NRP) zu erarbeiten, die unter anderem auf Strukturreformen und die Erhöhung der Forschungs- und Bildungsausgaben bis 2010 zielen. ${ }^{13}$ Seit Herbst 2006 werden Fortschritts- und Umsetzungsberichte dieser Reformstrategien erstellt und der Europäische Rat wird nun auf seiner Tagung im März 2007 eine erste Bilanz zur Umsetzung der reformierten Lissabonstrategie ziehen. Für die deutsche Ratspräsidentschaft wird es darauf ankommen, dass etwaige Defizite klar benannt und nach Möglichkeit auch Lösungsoptionen entwickelt werden. Was sich die Europäische Union angesichts der bisherigen Geschichte der Lissabon-Strategie nicht leisten kann, wäre ein ,Schönreden' der Probleme. Allerdings ist aufgrund des zu verzeichnenden (moderaten) wirtschaftlichen Aufschwungs, der sich auch 2007 fortzusetzen scheint, kein übermäßiger Problemhaushalt zu erwarten, so dass die deutsche Präsidentschaft hier ein Stück weit entlastet sein wird.

Nichtsdestotrotz sollte sich die deutsche Ratspräsidentschaft aktiv für die Weiterentwicklung der Lissabon-Strategie einsetzen und weiterführende Ansätze für die europäische Wachstums- und Beschäftigungsstrategie entwickeln. Das bedeutet auch, mit der Europäischen Kommission im Hinblick auf die neue Binnenmarktstrategie zusammenzuarbeiten, die von der Kommission vorgelegt wird ${ }^{14}$, um Europas Wettbewerbsfähigkeit durch einen einheitlichen geregelten Markt zu stärken, den es in vielen Bereichen wie Energie, Finanzdienstleistungen und Postdienstleistungen ${ }^{15}$ immer noch nicht gibt.

Aus Sicht der deutschen Ratspräsidentschaft geht es vor allem aber auch um eine Vielzahl von verschiedenen Maßnahmen, die erst in der Summe größere Effekte erzielen können wie die Verabschiedung der geplanten Roaming-Verordnung (um dem ,Abkassieren` bei der Mobilfunknutzung im Ausland einen Riegel vorzuschieben), ein stärkeres Rechtsschutzsystem bei öffentlichen Aufträgen, die Weiterentwicklung der Standardisierung bei Waren und

12 Vgl. Daniel Göler: Die Lissabon-Strategie. Ein europäischer Gestaltungsversuch?, in: Christoph Linzbach/ Uwe Lübking/Bernd Schulte/Stephanie Scholz (Hrsg.): Globalisierung und europäisches Sozialmodell, BadenBaden, im Erscheinen.

13 Eine Auflistung ist abrufbar unter: europa.eu/growthandjobs/pdf/nrp_2005_en.pdf (letzter Zugriff: 26.01. 2007).

14 Vgl. hierzu den Ergebnisbericht der öffentlichen Konsultationen zur Zukunft des Binnenmarktes vom Juni 2006, abrufbar unter: http://ec.europa.eu/internal_market/strategy/index_de.htm\#report (letzter Zugriff: 26.01. 2007).

15 Letzteres ist für Deutschland von Bedeutung, da das deutsche Postmonopol bei Briefen 2008 ausläuft. 
Dienstleistungen, die Förderung des Handels durch vermehrte gegenseitige Anerkennung von Zulassungs- und Produktvorschriften, die weitere Arbeit an dem geplanten Statut für die europäische Privatgesellschaft für KMU (kleine und mittlere Unternehmen), die Verhandlungen über die Richtlinie zur grenzüberschreitenden Sitzverlegung von Unternehmen und die verstärkte Umsetzung des Aktionsplanes für Finanzdienstleistungen. Zu Letzterem strebt die Präsidentschaft eine größere „Konvergenz im Aufsichtshandeln“ 16 an, geht hingegen nicht auf die Frage einer Europäischen Aufsichtsbehörde ein, wie sie von einigen starken (auch deutschen) Finanzdienstleistern immer wieder angesprochen wird. ${ }^{17}$ Ein wichtiges Anliegen der Ratspräsidentschaft besteht zudem in der Nivellierung von Wettbewerbsverzerrungen bei der Unternehmensbesteuerung. Hier bleibt aufgrund starker Widerstände bei etlichen Mitgliedstaaten jedoch nur der Weg, wenigstens eine Vereinheitlichung der Bemessungsgrundlagen anzustreben, wofür dann 2008 ein Legislativvorschlag der Kommission erwartet wird.

Besondere Akzente setzt die deutsche Ratspräsidentschaft im Transportsektor, was sowohl dem Handel auf dem Binnenmarkt wie auch dem grenzüberschreitenden Personenverkehr und damit einem bürgernahen Bereich zugute käme. So strebt die Bundesregierung substanzielle Ergebnisse bei den Luftverkehrsverhandlungen mit den USA an ${ }^{18}$ und will die Barrieren im europäischen Bahnverkehr abbauen, die Rechtsetzung zur Vollendung des europäischen Eisenbahnraumes voranbringen, die Beratungen über die Verordnung zu öffentlichen Personenverkehrsdiensten abschließen und das Satelliten-Navigationssystem GALILEO weiter aufbauen. ${ }^{19}$

Es geht aber nicht nur um die Vertiefung des Binnenmarktes zur Förderung von Industrie und Handel sowie zur günstigeren Angebotsversorgung der Bürger. Es geht auch um die Stärkung Europas im Kontext der Globalisierung durch einen funktionierenden Binnenmarkt und eine leistungsfähige europäische Währung (die mittlerweile die zweite Reservewährung nach dem Dollar ist) sowie damit verbunden den Einsatz des ökonomischen Gewichts Europas bei der Gestaltung und Beeinflussung des Welthandels. Zu letzterem gehören die Verbesserung der externen Schutzinstrumente gegen Dumping und unfaire Handelspraktiken, das Vorgehen gegen unerlaubte Subventionen und die Missachtung geistiger Eigentumsrechte, die Sicherstellung der europäischen Rohstoff- und Energieversorgung, der Ausbau bilateraler und multilateraler Handelsbeziehungen (Doha-Runde) und die Verabschiedung einer von der Kommission erneut vorzulegenden EU-Marktzugangsstrategie ${ }^{20}$ für europäische Waren, Dienstleistungen und Investitionen in Drittländern. Dies alles sind klassische Instrumente der EU zur Beeinflussung der Globalisierung - Instrumente, über die kein einzelner Mitgliedstaat der Europäischen Union verfügt und im Vergleich zur Größe des europäischen Wirkungspotenzials als einzelner auch gar nicht verfügen könnte.

Schwieriger zu lösen oder anzugehen sein wird hingegen die Frage des ,europäischen Sozialmodells'. Hauptkritikpunkt ist hier, dass sich Europa zunehmend am angelsächsischen Wirtschaftsmodell orientiere und die soziale Komponente vernachlässige, was nicht den (kontinental)europäischen Traditionen entspreche. Diese Kritik sticht zwar nicht richtig,

16 Vgl. Bundesregierung: Europa gelingt gemeinsam, S. 6.

17 Vgl. Friedrich Heinemann/Mathias Jopp: The benefits of a working European retail market for financial services, Bonn/Mannheim 2002.

18 Vgl. Bundesregierung: Europa gelingt gemeinsam, S. 11; seit 2003 werden Verhandlungen mit den USA über ein Luftverkehrsabkommen mit dem Ziel der Schaffung eines offenen Luftverkehrsraums geführt.

19 Vgl. ebenda, S. 12.

20 Vgl. Europäische Kommission: Das globale Europa - im Wettbewerb mit der Welt, 04.10.2006, abrufbar unter: http://ec.europa.eu/comm/trade/index_en.htm (letzter Zugriff: 26.01.2007). 
wenn die Umverteilungsleistungen der EU im Bereich der Regional-, Struktur- und Kohäsionspolitik sowie die Direktsubventionen an die Landwirtschaft berücksichtigt werden, die Binnenmarktprogrammatik hat aber dennoch eine Art „,neo-liberal bias“21. Dies ist vor allem darauf zurückzuführen, dass auf Marktbelebung durch Deregulierung gesetzt und europaweite Regulierungen, wie sie zum Aufbau einer transnationalen Sozialpolitik erforderlich wären, weitgehend vermieden werden. Fritz W. Scharpf hat diesen Mechanismus als Bevorzugung negativer Integration vor positiver Integration herausgearbeitet. ${ }^{22}$

Eines der Hauptprobleme im sozialen Bereich besteht in den völlig unterschiedlichen und in nationaler Verantwortung liegenden historisch gewachsenen Sozialpolitiken sowie in den für eine entsprechende europäische Variante erforderlichen enormen Aufwendungen, so dass bislang auf EU-Ebene sozialpolitische Maßnahmen nur in einer die nationale Sozialpolitik ergänzenden oder flankierenden Weise ergriffen wurden. Die deutsche Präsidentschaft verspricht sich zumindest von einer Ministerkonferenz, die Debatte über das europäische Sozialmodell mit konkreteren Inhalten füllen zu können, und will stärker auf die Überprüfung gerade der sozialen Auswirkungen europäischer Gesetzesvorhaben drängen. Wesentlich konkreter sind die geplante Intensivierung der Arbeiten an der europäischen Richtlinie zur Übertragbarkeit von Betriebsrenten für die Unterstützung bei grenzüberschreitender Arbeitnehmermobilität sowie die schrittweise Entwicklung einer Gemeinschaftsstrategie zu Gesundheit und Sicherheit am Arbeitsplatz. ${ }^{23}$ Außerdem will sich die Präsidentschaft in den bürgernahen Bereichen des Gesundheits- und Verbraucherschutzes engagieren und in diesem Zusammenhang den Verordnungsentwurf zu neuartigen Therapien vorantreiben, die Novellierung der Medizinproduktrichtlinie abschließen und sich für die Umsetzung der verbraucherpolitischen Strategie der Union einschließlich des dazugehörigen Aktionsprogramms (2007-2013) einsetzen. ${ }^{24}$

Da die Sozialpolitik vielfach als essentiell für die Akzeptanz des europäischen Integrationsprozesses angesehen wird, wäre es für die deutsche Ratspräsidentschaft und die europäischen Institutionen wichtig, die in den Verträgen existierenden, in der Öffentlichkeit aber wenig bekannten oder wahrgenommenen sozial- und beschäftigungspolitisch relevanten Regelungen zu verdeutlichen, einschließlich der in der Grundrechtecharta enthaltenen sozialen Grundrechte, der Ziele der europäischen Sozialagenda und des mit der finanziellen Vorausschau 2007 beschlossenen Globalisierungsfonds. Dieser stellt für die Wiedereingliederung von ,Globalisierungsarbeitslosen“ ab Januar 2007 jährlich immerhin wenigstens 500 Millionen Euro zur Verfügung. Die sozialpolitische Agenda 2006-2010 der EU legt die Rahmenprioritäten zur Weiterentwicklung des europäischen Sozialmodells als Bestandteil der Lissabon-Strategie fest. ${ }^{25}$ Der Schwerpunkt der Maßnahmen liegt in den Gebieten Beschäftigung, Qualifizierung sowie Chancengleichheit und soziale Eingliederung. Hierunter fallen unter anderem die Regelungen zur Ermöglichung der Mitnahme von Renten- und Sozialversicherungsansprüchen, die (Wieder-)Eingliederung von Frauen in den Arbeitsmarkt sowie die Aktualisierung des Arbeitsrechts. ${ }^{26}$

21 Vgl. Andrew Moravcsik: What Can We Learn from the Collapse of the European Constitutional Project?, in: Politische Vierteljahresschrift 2/2006, S.219-241, hier: S.230.

22 Fritz W. Scharpf: Balancing Positive and Negative Integration. The Regulatory Options for Europe, MPIfG Working Paper 97/8.

23 Vgl. Bundesregierung: Europa gelingt gemeinsam, S. 13.

24 Vgl. Ebenda.

25 Vgl. Rat der Europäischen Union: Europäische Sozialagenda 2000-2005, abrufbar unter: http://ue.eu.int/uedocs/cms_data/librairie/PDF/SocialAgenda_DE.pdf (letzter Zugriff: 26.01.2007).

26 Europäische Kommission: Mitteilung der Kommission-Sozialpolitische Agenda, KOM(2005)33 endgültig. 


\section{Die Entwicklung einer europäischen Energiepolitik}

Auffallend beim Thema Energiepolitik ist, dass es in den letzten 10 Jahren zwar ständig irgendwie auf der europäischen Agenda stand, aber immer unter wechselnden Vorzeichen behandelt wurde. So ging es Mitte der 90er Jahre im Rahmen der energiepolitischen Initiativen der Europäischen Union primär um den Aspekt der Marktliberalisierung. Mit der Verabschiedung des Kyoto-Protokolls und der in ihm festgelegten Treibhausgasreduzierungen trat zunehmend der Klimaschutz in den Vordergrund europäischer Regelsetzung. Spätestens mit der ukrainisch-russischen Energiekrise Anfang 2006, die auch für einige EU-Staaten zu kurzfristigen Lieferrückgängen führte, ${ }^{27}$ wurde dann verstärkt die Energieversorgungssicherheit priorisiert, was sich unter anderem am gemeinsamen Papier von Javier Solana und Benita Ferrero-Waldner zur Energieaußenpolitik zeigt. ${ }^{28}$ Die aktuellen Krisen in wichtigen Energielieferregionen wie im Mittleren Osten, aber auch der jüngste russisch-weißrussische Gas- und Ölstreit, der die vorübergehende Schließung der wichtigen Erdölpipeline ,Freundschaft" mit sich brachte, ${ }^{29}$ haben dazu beigetragen, dass die Energiepolitik insgesamt und insbesondere der Aspekt der strategischen Energiesicherheit noch stärker in den Fokus politischer Initiativen gerückt sind und dementsprechend einen Schwerpunkt der deutschen EURatspräsidentschaft darstellen. ${ }^{30}$

Erschwerend für eine europäische Energiepolitik ist bis heute, dass diese im wesentlichen über vertragsrechtliche, Hilfskonstruktionen` betrieben wird, das heißt über die Marktliberalisierung und die Wettbewerbspolitik, die Umweltpolitik sowie - in Teilbereichen - die Gemeinsame Außen- und Sicherheitspolitik; bei Fragen der Kernenergie steht noch der Rahmen des Euratom-Vertrages zur Verfügung. ${ }^{31}$ Dies führt zwangsläufig dazu, dass je nach Politikbereich mal eher der Aspekt der Wirtschaftlichkeit, mal der des Umweltschutzes beziehungsweise der Nachhaltigkeit und mal der der Versorgungssicherheit im Vordergrund stehen. Ein eigenes Energiekapitel gibt es in den bestehenden europäischen Verträgen - anders als im zur Zeit auf Eis liegenden Verfassungsvertrag ${ }^{32}$ - hingegen nicht.

An konkreten Initiativen steht für die deutsche Ratspräsidentschaft die Verabschiedung eines energiepolitischen Aktionsplans auf der Frühjahrstagung des Europäischen Rates an, ${ }^{33}$ der Grundlage für eine kohärente europäische Energiepolitik werden soll. Inhaltlich hat der Aktionsplan mehrere Dimensionen: längerfristige Zielvorgaben, wie die Reduktion von Treibhausgasen oder die Förderung erneuerbarer Energien, konkrete Rechtsetzungsprojekte im Bereich der Binnenmarktvollendung für den Energiesektor, Anstöße für internationale Initiativen und Verträge, die Entwicklung einer Energieaußenpolitik und die Koordinierung der nationalen Politiken. ${ }^{34}$ Bei der konkreten Ausarbeitung wird die deutsche Ratspräsidentschaft zu berücksichtigen haben, dass Aspekte wie Wirtschaftlichkeit, Umweltschutz/Nachhaltigkeit und Versorgungssicherheit in einem ausgewogenen Verhältnis zueinander stehen. ${ }^{35}$

27 Vgl. Enno Harks: Der russisch-ukrainische Gas-Streit, SWP-Diskussionspapier FG 8 2006/01.

28 Vgl. Papier der Kommission und des Generalsekretärs/Hohen Vertreters für den Europäischen Rat: Eine Aussenpolitik zur Förderung der EU-Interessen im Energiebereich, S160/06.

29 Vgl. Renata Goldirova: EU frets about gas risks after oil disruptions, in: euobserver.com, 11.012007.

30 Vgl. Bundesregierung: Europa gelingt gemeinsam, S. 9.

31 Sebastian Wolf: Die Zukunft des Euratom-Vertrags, in: integration 4/2006, S. 297-302.

32 Vgl. Vertrag über eine Verfassung für Europa, Teil III, Titel III, Kapitel III, Abschnitt 10.

33 Vgl. Europäischer Rat: Schlussfolgerungen des Vorsitzes, 15./16. Juni 2006, S. 10.

34 Vgl. ebenda, S. 11.

35 Vgl. Gerd Eisenbeiß: Energie der Zukunft - Zukunft der Energien, in: Peter Fischer/Klaus Wiegandt: Die Zukunft der Erde. Was verträgt unser Planet noch? Frankfurt am Main 2005, S. 159 - 181, hier: S. 159-165. 
Was den Aspekt der Wirtschaftlichkeit anbelangt, wird der Schwerpunkt der deutschen Ratspräsidentschaft bei der Vollendung des Energiebinnenmarktes im Strom und Gassektor liegen. Hierzu bedarf es eines neuen Anschubs schon allein mit Blick auf die Tatsache, dass etliche der bereits verabschiedeten Richtlinien noch nicht gänzlich umgesetzt sind. Auch gilt es aus der Perspektive des Wettbewerbsrechts, die Frage des Umgangs mit versorgungstechnischen Oligopolen ${ }^{36}$ und die von der Kommission vorgeschlagene Trennung von Netz und Betreiber mit dem Ziel der Senkung der Gas- und Strompreise für die Bürger zu klären. ${ }^{37}$ Die Bundesregierung und die deutsche Energiewirtschaft haben allerdings bereits deutlich gemacht, dass sie einer zwangsweisen Trennung von Netz und Betreibern ablehnend gegenüber stehen; Gleiches gilt für die Vorschläge zu einem europäischen Regulator im Energiesektor. ${ }^{38}$ Die Bundesregierung setzt statt dessen auf eine engere Koordinierung der verschiedenen nationalen Regulierungsbehörden und möchte das europäische Öl- und Gaspipelinenetz sowie das Verbundnetz im Strombereich ausbauen, wozu vor allem die Zahl der Grenzkoppelstellen zu erhöhen wäre. Denn durch europaweite Netze wird nicht nur der Wettbewerb, sondern auch die Versorgungssicherheit und die Solidarität zwischen den Mitgliedstaaten erhöht, da kurzfristige Lieferengpässe in bestimmten Regionen schneller ausgeglichen werden können.

Auch beim Umwelt- und Klimaschutz wird die deutsche Ratspräsidentschaft energiepolitisch aktiv werden, ${ }^{39}$ denn der Klimawandel ist die zentrale Herausforderung des 21. Jahrhunderts. Deshalb sollen erste Initiativen für ein post-Kyoto-Protokoll ab 2012 ergriffen werden. ${ }^{40}$ Zwar ist die Europäische Union bisher weltweit führend in der Reduktion von Treibhausgasen, bei einigen Mitgliedstaaten bestehen aber Zweifel am Erreichen der KyotoReduktionsziele bis 2012 und umweltpolitische Verbände halten eine weit höhere Emissionsreduktion für erforderlich. ${ }^{41}$ Auch muss das zum 1. Januar 2005 eingeführte System der Treibhausgasemissionszertifikate erst noch zu einer wirklichen Reduzierung der Treibhausgase genutzt werden. Hierbei stehen die nationalen Allokationspläne für Emissionen im Zeitraum 2008-2012 im Mittelpunkt, die zwar von den jeweiligen Mitgliedsländern aufzustellen sind, von der Kommission aber abgelehnt werden können. ${ }^{42}$ Zurzeit zeichnet sich hier eine Auseinandersetzung zwischen der Bundesregierung und der Kommission ab, da letztere auf eine deutlich höhere Treibhausgasreduktion dringt als vom zuständigen Bundesministerium vorgeschlagen. ${ }^{43}$

Im Bereich der Energieeffizienz sind noch erhebliche Potenziale vorhanden, wie der von der Kommission vorgelegte Aktionsplan für Energieeffizienz ${ }^{44}$ ausführt, der die Einsparmöglichkeiten bis 2020 auf 20 Prozent beziffert. ${ }^{45}$ Die Bundesregierung möchte diese Initiative während der deutschen Ratspräsidentschaft besonders aufgreifen, denn die Steigerung der Energieeffizienz ist nicht nur umweltpolitisch geboten, sondern trägt auch zur Verbesse-

36 Was auch Gegenstand der geplanten Kartellrechtsnovellierung von Wirtschaftsminister Glos sein soll. Vgl. Nils-Viktor Sorge: Ins Netz gegangen, in: Der Tagesspiegel, 12.01.2007, S. 2.

37 Vgl. Europäische Kommission: An Energy Policy for Europe, COM(2007)1, S. 7.

38 Ebenda, S. 8.

39 Vgl. Bundesregierung: Europa gelingt gemeinsam, S. 9.

40 Vgl. Rat der Europäischen Union: Achtzehnmonatsprogramm, S. 51.

41 Vgl. Greenpeace Deutschland: Klimaschutz im Schneckentempo. Resümee der Klimaschutzkonferenz im November 2006 in Nairobi, abrufbar unter: http://www.greenpeace.de (letzter Zugriff: 26.01.2007).

42 Vgl. Richtlinie 2003/87/EG des Europäischen Parlaments und des Rates vom 13.10.2003.

43 Vgl. Spiegel Online: EU fordert von Deutschland noch mehr CO2-Einsparung, 29.11.2006.

44 Vgl. Europäische Kommission: Aktionsplan für Energieeffizienz. Das Potential ausschöpfen, KOM(2006)545 endgülitig.

45 Ebenda, S. 6. 
rung der Versorgungssicherheit und Wirtschaftlichkeit bei, da die sicherste und billigste Energie diejenige ist, die nicht verbraucht wird.

Die Förderung umweltfreundlicher Energieträger ist der zweite Schlüssel zu einer nachhaltigen Energiepolitik, wobei es hier unterschiedliche Ansätze gibt: die stärkere Förderung von erneuerbaren Energien wie Wind, Wasser, Sonne, Biomasse und Biotreibstoff, die Einlagerung von $\mathrm{CO}_{2}$ aus der Verbrennung fossiler Energieträger (Clean-Coal-Energie) und $\mathrm{CO}_{2}$-freie Energiegewinnungsverfahren wie die Kernfusion und Kernspaltung. Der Energieaktionsplan wird hierbei eine ausgewogene Förderung verschiedener Energieträger umfassen, sei es als Zielvorgabe für künftige Rahmenforschungsprogramme oder durch Festlegung eines Mindestanteils von $\mathrm{CO}_{2}$-freien und erneuerbaren Energien. Besondere Chancen ergeben sich für die deutsche Ratspräsidentschaft im Bereich Klima- und Umweltschutz auch dadurch, dass sie die entsprechenden europäischen Initiativen aufgrund der ebenfalls von ihr im ersten Halbjahr 2007 wahrgenommenen G8-Präsidentschaft in einen globalen Rahmen stellen beziehungsweise enge Abstimmungen mit den größten Industrienationen im Bereich Klima- und Umweltschutz vornehmen kann.

Bei der Versorgungssicherheit liegt das strategische Problem der EU darin, dass die schon heute hohe Abhängigkeit von Energieimporten sich in den nächsten 20 bis 30 Jahren aufgrund der zur Neige gehenden europäischen Öl- und Gasvorkommen nochmals deutlich auf circa 70 Prozent erhöhen wird. ${ }^{46}$ Verkompliziert wird die Situation dadurch, dass der überwiegende Teil der europäischen Öl- und Gasimporte aus dem Mittleren und Nahen Osten, Zentralasien und Russland stammt, alles Regionen beziehungsweise Länder, die politisch problematisch sind oder wie Russland nicht davor zurückschrecken, Energielieferungen als politisches Druckmittel gegenüber Nachbarstaaten und Transitländern einzusetzen. ${ }^{47}$

Ob für eine kohärente europäische Energieaußenpolitik neue institutionelle Einrichtungen notwendig werden, wie der vom französischen Ministerpräsidenten de Villepin vorgeschlagene Posten eines „Représentant spécial pour l'énergie“, ${ }^{48}$ der von deutscher Seite zumindest inoffiziell als interessante Idee bezeichnet wurde, ${ }^{49}$ bleibt abzuwarten. Bei allen Diskussionen um die strategische Energiesicherheit gilt es jedoch zu berücksichtigen, dass es zu eindimensional wäre, die Energieversorgungssicherheit nur in geostrategischer Perspektive als „Wettlauf“ um sichere Energiequellen und Transportwege zu sehen. ${ }^{50}$ So wichtig diese Ansätze sein mögen, es muss auch um den Export von Demokratie und marktwirtschaftlichen Strukturen gehen, die auch im Energiebereich eine größere Sicherheit bieten würden. Die Gründung der Europäischen Energiegemeinschaft zwischen der EU und den Staaten Südosteuropas ${ }^{51}$ ist hierzu ein erster wichtiger Schritt, zumal wenn diese - wie von der deutschen Ratspräsidentschaft beabsichtigt - auf weitere Staaten ausgedehnt werden

46 Vgl. Europäische Kommission: Grünbuch. Eine europäische Strategie für nachhaltige, wettbewerbsfähige und sichere Energie, KOM (2006)105 endgültig, S. 3.

47 Vgl. Friedemann Müller: Global Energy Resource Supply. Strategic Trends, SWP Working Paper FG 8 2006/ 03, S. 4.

48 Dominique de Villepin: Allocution du Premier Ministre au 10e forum international de la fondation Bertelsmann, 22.09.2006, abrufbar unter: www.premier-ministre.gouv.fr (letzter Zugriff: 26.01.2007).

49 So Staatssekretär Joachim Wuermeling auf der Konferenz „Energy security in an uncertain world“, 05.10.2006 in Berlin.

50 Eine kritische Auseinandersetzung hierzu findet sich bei: Kristina Kurze: Mapping Securitisation Dynamics in the European Union. The Case of an emerging Energy Policy for Europe, Paper presented at the "Security Theory - Critical Innovations" Seminar 27.11.-1.12.2006, Universität Kopenhagen.

51 Diese wurde geschlossen zwischen der Europäischen Gemeinschaft und Albanien, Bulgarien, Bosnien-Herzegovina, Kroatien, Mazedonien, Montenegro und Serbien. Vgl. Treaty establishing the Energy Community, abrufbar unter: http://www.seerecon.org/infrastructure/sectors/energy/documents/Treatyenergy.pdf (letzter Zugriff: 26.01.2007). 
sollte. ${ }^{52}$ Kommt noch die von Außenminister Steinmeier angekündigte europäische Zentralasienstrategie hinzu ${ }^{53}$, in der auch die Energiepolitik einen wichtigen Bestandteil bilden würde, so könnten die Transportwege bis in den zentralasiatischen Raum gesichert werden. Schließlich gilt es auch den Energiedialog mit großen Verbraucherländern wie China, Indien und den USA zu intensivieren und die Energiebeziehungen in das neue Partnerschafts- und Kooperationsabkommen mit Russland aufzunehmen. ${ }^{54}$

Untrennbar verbunden mit der Frage der Versorgungssicherheit ist die Binnensolidarität der EU-Staaten. Während diese Frage bei Erdöl durch die strategischen Reserven im Rahmen der Internationalen Energieagentur (IEA) geregelt ist, ist sie bei Erdgas noch offen. Denn die Anlage der Notvorräte liegt in der Verantwortung der Unternehmen, so dass es in Europa völlig unterschiedliche Notreserven gibt, wobei Deutschland die größten Kapazitäten besitzt. Nicht zuletzt deshalb spricht sich die Bundesregierung hier zwar für eine bessere Transparenz und Bestandsaufnahme aus, ist bislang aber gegen eine ,Europäisierung ' von Notreserven bei Gas. Ebenfalls abgelehnt werden europäische Vorgaben für den Energiemix. Zwar ist es unbestritten, dass eine sichere Energieversorgung nur auf der Basis einer Diversifizierung von Herkunftsländern, Transportwegen und auch Energieträgern gewährleistet werden kann. Allerdings einigten sich die Staats- und Regierungschefs auf deutsches Drängen hin bereits im Juni 2006 darauf, dass der Energieaktionsplan den nationalen Energiemix und damit auch die Frage des Anteils der Nuklearenergie unberührt lassen soll. ${ }^{55}$

Bei all diesen Punkten wird die zentrale Frage für die europäische Energiepolitik sein, ob es den Willen gibt, die Europäische Union mit einer eigenen energiepolitischen Kompetenz auszustatten. Insoweit wäre für die deutsche Präsidentschaft zu prüfen, ob der energiepolitische Aktionsplan nicht auch schon die Möglichkeiten zu primärrechtlichen Veränderungen im Bereich der Energiepolitik thematisieren sollte, auf die dann bei der Wiederaufnahme des Verfassungsprozesses oder der Verabschiedung eines verfassungsähnlichen Vertrags zurückgegriffen werden könnte.

Insgesamt lassen sich die Präferenzen der deutschen Ratspräsidentschaft für den im März zu verabschiedenden Energieaktionsplan dahingehend zusammenfassen, dass zunächst die Vollendung des Binnenmarktes für Strom und Gas bis Juli 2007 angestrebt werden soll, wozu auf eine konsequente Umsetzung der bestehenden europäischen Vorgaben und die Förderung des Wettbewerbs im Energiesektor hingearbeitet werden muss. Zudem möchte die Bundesregierung im Energieaktionsplan einen klaren Schwerpunkt auf den Umwelt- und Klimaschutz legen. Um erste konkrete Zielvorgaben für eine post-Kyoto-Vereinbarung anzugehen, wird sie besonderen Wert auf die Steigerung der Energieeffizienz und den Ausbau erneuerbarer Energien legen, wobei zum einen die Priorität im Gebäudesektor und zum anderen im Kraftstoffsektor liegt. Und schließlich wird sich die Bundesregierung dafür einsetzen, dass der Aktionsplan auf klare Perspektiven für energiepolitische Partnerschaften mit Lieferanten-, Transit- und Verbraucherländern drängt.

52 Vgl. Michael Glos: Energiepolitik während der deutschen EU-Ratspräsidentschaft, in: integration 1/2007, S. 50-53; Frank-Walter Steinmeier: Gemeinsam die Energieversorgung sichern, in: Handelsblatt, 23.03.2006.

53 Vgl. Die Friedensverantwortung der Europäischen Union am Beginn der deutschen Präsidentschaft - Interview mit Bundesaußenminister Steinmeier, in: Berliner Morgenpost, 24.12.2006.

54 Vgl. Günter Gloser: Energiepolitik für Europa - ein Schwerpunkt der deutschen Ratspräsidentschaft, in: integration 4/2006, S. 315-317, hier: S. 317.

55 Vgl. Europäischer Rat: Schlussfolgerungen des Vorsitzes, 15./16. Juni 2006, S. 11. 


\section{Die Innere Sicherheit der EU und europäische Migrationspolitik}

Im Verlauf der Ratifizierungsdebatten zum Verfassungsvertrag in den Mitgliedstaaten wurde deutlich, dass die Bürger ein effektives Handeln der EU in den für sie wichtigen Alltagsfragen anmahnen. ${ }^{56}$ In diesem Zusammenhang wird immer wieder die innere Sicherheit genannt, wobei es um internationalen Terrorismus, grenzüberschreitende Kriminalität und den Bereich der Einwanderung, insbesondere das Problem der illegalen Migration geht. ${ }^{57}$ Wie wichtig den Menschen gerade diese drei Themen der Innen- und Justizpolitik sind, zeigt sich auch an deren Nennung in Meinungsumfragen unter den von den Bürgern empfundenen sieben wichtigsten ,Sorgen“. 58

Vor allem nach den Tragödien von London und Madrid und den glücklicherweise fehlgeschlagenen Anschlägen auf deutsche Züge im Sommer letzten Jahres hat die Problematik des internationalen Terrorismus auch in Europa an Bedeutung gewonnen. Es war aber schon kurz nach den Terroranschlägen in den USA vom 11. September 2001 zu einer Intensivierung der sich ohnehin relativ dynamisch entwickelnden innen- und justizpolitischen Zusammenarbeit zwischen den EU-Mitgliedstaaten gekommen, ${ }^{59}$ die in verschiedenen Maßnahmen wie etwa den Europäischen Haftbefehl mündeten, der seit August 2006 mit dem Inkrafttreten des überarbeiteten Gesetzes zur Umsetzung des Europäischen Haftbefehls (EUHbG II) nunmehr auch in Deutschland vollstreckt werden kann. Zudem wurden der grenzüberschreitende Austausch von Daten zur Verbrechens- und Terrorismusbekämpfung vereinfacht, EUROPOL ausgebaut und die Zusammenarbeit bei der Kontrolle der Außengrenzen verbessert. Schließlich schlossen Deutschland, Frankreich, Spanien, Österreich und die Benelux-Staaten im Mai 2005 nach dem Vorbild des Schengener Abkommens den sogenannten Vertrag von Prüm zur Vertiefung der grenzüberschreitenden Zusammenarbeit bei der Terrorismusabwehr, der Bekämpfung der internationalen Kriminalität und bei Fragen der illegalen Immigration ${ }^{60}$, wobei hauptsächlich auf erleichterten Austausch von Daten und den gegenseitigen Zugriff auf diese wie auch die intensivere operative Zusammenarbeit zwischen den Vertragsstaaten gesetzt wird.

Die deutsche Ratspräsidentschaft möchte den Vertrag von Prüm in den EU-Rahmen überführen, da sich die Vertragspartner in einer Vorreiterrolle für die Entwicklung der inneren Sicherheit in der EU sehen. Eine geeignete Möglichkeit hierfür bestünde in der Nutzung der verstärkten Zusammenarbeit nach den Artikeln 40 - 40b sowie 43 - 45 EUV, wobei es allerdings wegen der vielen souveränitätspolitisch und datenrechtlich begründeten Vorbehalte etlicher Mitgliedstaaten nicht einfach sein dürfte, die erforderliche qualifizierte Mehrheit für die Autorisierung einer verstärkten Zusammenarbeit zu allen Materien des Prümer Vertrages zu erreichen. Könnte eine Überführung in den EU-Rahmen aber gelingen, wäre dies von großer Bedeutung im Hinblick auf die Wahrung und Schaffung der Einheitlichkeit des europäischen Rechtsraumes und die Herstellung der Kompatibilität des Prümer Vertrages mit existierenden rechtlichen und institutionellen Vorkehrungen der EU.

56 Vgl. Moravcsik: What Can We Learn, S. 219-241.

57 So Peter Altmaier, Parlamentarischer Staatssekretär im Bundesministerium des Innern in einem IEP-Mittagsgespräch am 23. Mai 2006 in Berlin.

58 Europäische Kommission: Eurobarometer 65. Die öffentliche Meinung in der Europäischen Union. Erste Ergebnisse, Frühjahr 2006, S. 8.

59 Vgl. Jörg Monar: Die EU als Raum der Freiheit, der Sicherheit und des Rechts und die Herausforderung des internationalen Terrorismus, in: integration 3/2002, S. 171-186, hier: S. 171.

60 Vgl. hierzu Daniela Kietz/Andreas Maurer: Der Vertrag von Prüm: Vertiefungs- und Fragmentierungstendenzen in der Justiz- und Innenpolitik der EU, integration 3/2006, S. 201-212. 
Zur Bekämpfung von Terrorismus, organisierter Kriminalität sowie Drogen- und Menschenhandel setzt die deutsche Ratspräsidentschaft auf eine Weiterentwicklung des Rechtsrahmens für EUROPOL und größere Zuständigkeiten der Behörde (zum Beispiel durch Umwandlung in eine EU-Agentur). Zudem will sie sich für die Übertragung von operativen Befugnissen auf Gastbeamte in anderen Mitgliedstaaten und die Möglichkeit der polizeilichen Nacheile im Schengen-Raum einsetzen. Letztere wäre für eine bessere grenzüberschreitende Verfolgung von Straftätern von Vorteil. Die Frage der operativen Befugnisse von Polizeibeamten eines Mitgliedstaates in einem anderen Mitgliedstaat stößt aber aufgrund des jeweiligen nationalen Staatsrechtsverständnisses immer noch auf Widerstände.

Im Bereich der Asyl- und Migrationspolitik will die deutsche Präsidentschaft die Europäische Kommission beim Aufbau eines Visa-Informationssystems (VIS) unterstützen, um vor allem illegale Einwanderung und Visamissbrauch besser aufzudecken beziehungsweise zu verhindern. Zudem soll die Harmonisierung bei der Nutzung biometrischer Daten vorangetrieben und auf den Abschluss der zäh laufenden Verhandlungen über Rücknahmeabkommen mit Drittstaaten gedrängt werden. Fortgesetzt werden soll auch die Diskussion über die legale Einwanderung, zu der die Europäische Kommission eine Reihe von Legislativvorschlägen (Richtlinien) für Wirtschaftsimmigranten und die Einreise sowie den Aufenthalt hoch qualifizierter Arbeitskräfte vorbereitet. ${ }^{61}$ Hierbei könnte der deutsch-französische Vorschlag für ein Konzept der kontrollierten und zeitlich befristeten Einwanderung weiterentwickelt werden, einschließlich der Meldung von legalen Einwanderungsquoten seitens der Mitgliedstaaten und Visaerleichterungen unter bestimmten Umständen. ${ }^{62}$ Hinter dem Konzept der zirkulären Migration steht vor allem die Einsicht, dass mit Blick auf die demografische Entwicklung in Europa Einwanderer für bestimmte Segmente des europäischen Arbeitmarktes gebraucht werden und zudem durch die beachtlich großen Finanztransfers von Migranten in ihre Heimatländer ein bedeutender Teil indirekter Entwicklungshilfe geleistet wird. Vor diesem Gesamthintergrund will sich die deutsche Ratspräsidentschaft auch im Rahmen einer Konferenz der zuständigen Minister der Mitgliedstaaten mit den drängenden Fragen der besseren Integration von Zuwanderern beschäftigen. ${ }^{63}$

Andererseits möchte die deutsche Ratspräsidentschaft zur Abwehr illegaler Einwanderung und zur Bekämpfung von Terrorismus und transnationaler Kriminalität den Außengrenzschutz der EU verbessern, sich für eine möglichst baldige Inbetriebnahme des Schengen-Informationssystems II (SIS II) einsetzen und die Grenzschutzagentur FRONTEX, welche die operative Zusammenarbeit der Mitgliedstaaten beim Schutz der Außengrenzen koordiniert und unterstützt, personell aufstocken. Dabei geht es vor allem um die Schaffung eines Pools von Experten gegen illegale Einwanderung und die Übertragung von exekutiven Befugnissen auf Gastbeamte bei grenzpolizeilichen Kontrollen. ${ }^{64}$

Ein weiterer Zielbereich besteht in der Schaffung eines Einheitlichen Europäischen Rechtsraums parallel zum Binnenmarkt. Nach wie vor ist der Europäische Rechtsraum durch eine große Fragmentierung gekennzeichnet, was sich in vielfältiger Weise bemerkbar macht, wie zum Beispiel im unterschiedlichen Ehe-, Scheidungs-, Unterhalts- und Erb-

61 Vgl. Europäische Kommission: Legislativ- und Arbeitsprogramm, S. 14.

62 Beim Innenministertreffen in Stratford-upon-Avon hat Bundesinnenminister Wolfgang Schäuble (CDU) gemeinsam mit seinem französischen Amtskollegen Nicolas Sarkozy Vorschläge zu einer gemeinsamen EU-Einwanderungspolitik gemacht. Vgl. Hannelore Crolly: Schäuble fordert EU-Asylbehörde, in: Die Welt, 27.10. 2006.

63 So der Parlamentarische Staatssekretär des BMI, Peter Altmaier, auf der IEP-Konferenz zur deutschen Ratspräsidentschaft am 1. Dezember 2006 im Europasaal des Auswärtigen Amtes.

64 Vgl. Bundesregierung: Europa gelingt gemeinsam, S. 18. 
schaftsrecht, in Unzulänglichkeiten beim Schutz geistigen Eigentums, in Unterschiedlichkeiten beim Strafrecht und auch beim Verbraucherrechtsschutzniveau. Schließlich geht es um Probleme der gegenseitigen Anerkennung justizieller Entscheidungen selbst hinsichtlich des Europäischen Haftbefehls oder der Vollstreckung von Geldstrafen. ${ }^{65}$ Um deshalb im Bereich des Zivil- und Strafrechts den Binnenmarkt nicht im Zustand eines ,unvollendeten Stückwerks" 66 zu belassen, will sich der deutsche Vorsitz für die Vereinbarung von Mindeststandards in Strafverfahren einsetzen, die Verhandlungen über einen Rahmenbeschluss zu Mindestanforderungen an Rechte von Beschuldigten und Angeklagten zum Abschluss bringen, sich für eine Mindestdefinition von Listendelikten mit präzisierten Tatbeständen zur gegenseitigen Anerkennung gerichtlicher Entscheidungen engagieren, die Vernetzung der Strafregister über Frankreich, Spanien, Deutschland und Belgien hinaus ausweiten, das Projekt ,Work on e-justice' zum Informationsaustausch bei grenzüberschreitenden Justizverfahren vorantreiben und die festgefahrenen Verhandlungen über einen Rahmenbeschluss zur Mindestharmonisierung der Strafvorschriften bei rassistischen und fremdenfeindlichen Äußerungen wieder aufnehmen. Außerdem möchte die deutsche Präsidentschaft die Verordnungen zum Recht bei vertraglichen wie auch außervertraglichen Schuldverhältnissen (zum Beispiel Verkehrsunfällen) voranbringen beziehungsweise verabschieden. ${ }^{67}$

Während sich die letztgenannten Maßnahmen überwiegend auf die Stärkung der Bürgerrechte im EU-Binnenmarkt konzentrieren, lässt sich bei der Reaktion auf die neue Bedrohungslage nach dem 11. September 2001 ein Spannungsverhältnis zwischen Freiheitsrechten und innerer Sicherheit feststellen. Dies zeigt sich nicht zuletzt daran, dass Datenschutz oder parlamentarische Kontrolle bei intergouvernementalen Maßnahmen im Bereich Justiz und Inneres zur Verbrechensbekämpfung oder Terroristenabwehr hinterherhinken. So ist es weitgehend ungeklärt, wer beispielsweise für die ,neuen zwischenstaatlichen Vernetzungen “ nationaler Polizei- und Sicherheitsdienste und auch den Datenschutz an zentraler Stelle verantwortlich ist. Nationale Parlamente und Datenschutzbeauftragte können grenzüberschreitende Strukturen nur partiell kontrollieren und den europäischen Institutionen wie zum Beispiel auch dem Europäischen Datenschutzbeauftragten fehlt es an entsprechenden Kompetenzen, solange es sich nicht um Rechtsakte der Gemeinschaft handelt, die sich auf personenbezogene Daten beziehen. Wichtig wären zudem der Ausbau der Kontrollrechte des Europäischen Parlaments und auch der Befugnisse des Europäischen Gerichtshofs ${ }^{68}$. Es zeigt sich deshalb gerade auch hier die Bedeutung einer baldigen und erfolgreichen Wiederaufnahme des Europäischen Verfassungsprozesses, um durch die Stärkung der europäischen Institutionen und die Vergemeinschaftung der verbliebenen Teile der Innen- und Justizpolitik nicht nur eine effizientere EU-weite Politik in diesem Bereich zu erzielen, sondern auch bessere Transparenz und grenzübergreifende Kontrollmöglichkeiten zum Schutze der bürgerlichen Freiheitsrechte zu gewährleisten.

65 Peter-Christian Müller-Graff/Friedemann Kainer: Raum der Freiheit der Sicherheit und des Rechts, in: Werner Weidenfeld/Wolfgang Wessels (Hrsg.): Europa von A bis Z. Taschenbuch der europäischen Integration, Baden-Baden 2006, S. 332.

66 Vgl. Bundesregierung: Europa gelingt gemeinsam, S. 17.

67 Agence Europe: Bulletin Quotidien Europe, Nr. 9337, 05.01.2007, S. 6.

68 Eine Ausweitung der Kompetenzen des Europäischen Gerichtshofes im Rahmen der Terrorismusbekämpfung hat der Rat in seiner Sitzung am 4./5. Dezember 2006 in Brüssel befürwortet. Vgl. Rat der Europäischen Union: Mitteilung an die Presse, Tagung des Rates Justiz und Inneres, 4./5. Dezember 2006, Press 341, Nr: 15801/06, S.9. 


\section{Erweiterungs- und Nachbarschaftspolitik}

Durch den zum Januar 2007 erfolgten Beitritt Rumäniens und Bulgariens ist die jüngste Erweiterungswelle der Europäischen Union zu einem vorläufigen Abschluss gekommen. ${ }^{69}$ Mit Kroatien wird allerdings im üblichen Tempo systematisch weiter über den Beitritt des Landes zur EU verhandelt. Die übrigen Staaten des westlichen Balkans haben ebenfalls Beitrittsoptionen, deren Konkretisierung ist aber auf absehbare Zeit nicht zu erwarten. ${ }^{70}$ Mit Mazedonien ${ }^{71}$ und Albanien müssen zunächst einmal die Stabilisierungs- und Assoziierungsabkommen (SAA) implementiert werden, mit Bosnien-Herzegowina sind die entsprechenden Verhandlungen noch nicht abgeschlossen, ${ }^{72}$ und mit Serbien können sie erst weitergeführt werden, wenn das Land der Forderung der EU nachkommt, besser mit dem internationalen Kriegsverbrechertribunal zu kooperieren. ${ }^{73}$ Mit der Türkei laufen die Beitrittsverhandlungen zwar durch die Öffnung von Kapiteln, für die die technischen Voraussetzungen erfüllt sind, weitere, wichtige Kapitel zum Handel mit Gütern oder Dienstleistungen, zur Agrarpolitik, Fischerei- oder Transportpolitik, Zollunion oder Auswärtige Beziehungen werden aber nach hinten verschoben und keinerlei Verhandlungskapitel vorläufig geschlossen, solange sich die Türkei nicht in der Zypern-Frage bewegt. ${ }^{74}$ Während der deutschen Ratspräsidentschaft sind hier keine Veränderungen der Positionen der EU und insbesondere nicht der Türkei zu erwarten, in der in diesem Jahr zunächst die Präsidentschafts- und später dann die Parlamentswahlen anstehen. ${ }^{75}$

Erforderlich ist nach dem Abschluss der großen Erweiterung unter Einschluss Bulgariens und Rumäniens zunächst eine Phase der Konsolidierung der Europäischen Union, in der es eines wirksamen Vertiefungsschrittes bedarf beziehungsweise der Lösung der Verfassungskrise, um Handlungsfähigkeit, Effizienz und Legitimität der Europäischen Union zu stärken, bevor unter Berücksichtigung der Integrationsfähigkeit der EU nächste Schritte in der Erweiterungspolitik erfolgen können. ${ }^{76}$ Dies ist gleichermaßen aus funktionalen Gründen, wie aus Rücksicht auf die ,Erweiterungsmüdigkeit“ in weiten Teilen der EU-Bevölkerung erforderlich. Der Binnenmarkt in den neuen Mitgliedstaaten muss erst einmal vollständig umgesetzt und der gesamte EU-Rechtsbestand dort wirkungsvoll implementiert werden. Dies gilt ebenso für das Zusammenwachsen im Bereich des außen- und sicherheitspolitischen Acquis.

Während dieser Phase der internen Konsolidierung gilt es, Strategien und Optionen zur Stabilisierung des geopolitischen Umfelds der EU zu schaffen. Nach Auffassung der Bundesregierung muss deshalb die Europäische Nachbarschaftspolitik (ENP) ausgebaut werden, damit ,an den Außengrenzen der EU kein neuer, eiserner Vorhang ‘ errichtet“ ${ }^{\text {‘77 }}$ wird. Trotz

69 Zur Bilanz der Osterweiterung vgl.: Barbara Lippert (Hrsg.): Bilanz und Folgeprobleme der EU-Erweiterung, Baden-Baden 2004.

70 Vgl. Franz-Lothar Altmann: EU-Erweiterungsmüdigkeit und Westlicher Balkan, SWP-Aktuell 2005/A 60.

71 Auch Mazedonien hat den Status eines Beitrittskandidaten seit Dezember 2005.

72 Zeitpunkt der Aufnahme der Verhandlungen für den Abschluss eines Stabilisierungs- und Assoziierungsabkommens mit Bosnien-Herzegowina war November 2005. Diese sind zum jetzigen Zeitpunkt noch nicht abgeschlossen. Siehe dazu: http://ec.europa.eu/enlargement (letzter Zugriff: 26.01.2007).

73 Vgl. hierzu: http://ec.europa.eu/enlargement/serbia/eu_serbia_relations_en.htm (letzter Zugriff: 26.01.2007).

74 Rat der Europäischen Union: Tagung des Rates Allgemeine Angelegenheiten und Außenbeziehungen, 11.12.2006, Press 352, Nr. 16291/06, S. 8.

75 Zur aktuellen Situation vgl. Heinz Kramer: Türkei-Verhandlungen vor dem Aus? SWP-Aktuell, 2006/A 51.

76 Vgl. Europäische Kommission: Erweiterungsstrategie und wichtigste Herausforderungen für den Zeitraum 2006 - 2007 mit Sonderbericht über die Fähigkeit der EU zur Integration neuer Mitglieder, KOM(2006)649 endgültig, S. 18 .

77 Vgl. Erklärung des Bundeskabinetts zur deutschen EU-Ratspräsidentschaft, 05.11.06, abrufbar unter: www.bundesregierung.de (letzter Zugriff: 26.01.07). 
verschiedener Verbesserungsanläufe krankt die Nachbarschaftspolitik noch an einem geschlossenen Gesamtkonzept, an ausreichenden Finanzmitteln und deren besseren Verteilung zwischen den östlichen und südlichen ENP-Staaten. Zudem sind die Exportmöglichkeiten einiger ENP-Länder durch Beschränkungen der EU bei Agrarprodukten und Textilien begrenzt. Vor allem ist noch nicht ersichtlich, welche Perspektiven Ländern angeboten werden könnten, die eigentlich eine Vollmitgliedschaft in der EU anstreben.

Unabhängig davon gilt es zunächst, die Partnerschafts- oder Assoziierungsabkommen umzusetzen oder auch neu auszuhandeln ${ }^{78}$ und die Aktionspläne zu verbessern oder in einigen Fällen erst zu beschließen. In einem späteren Schritt können dann unter bestimmten Bedingungen Nachbarschaftsabkommen geschlossen werden, die die Beziehungen auf eine höhere Stufe stellen. Optionen bestehen in jedem Fall in der Verbesserung der Handels- und Wirtschaftsbeziehungen und der schrittweisen Heranführung an den Binnenmarkt, in engeren Kooperationen in bestimmten Politikbereichen wie in der Energie- oder Umweltpolitik und in der schrittweisen Einbeziehung der ENP-Länder in die Schengen-Politik sowie in die Bekämpfung des Terrorismus und der organisierten Kriminalität. Auch ist an die Zusammenarbeit bei bestimmten Aspekten der europäischen Außen- und Sicherheitspolitik zu denken, insbesondere an die Unterstützung bei der Lösung regionaler Konflikte und bei der Verbesserung der regionalen Zusammenarbeit. ${ }^{79}$

Die Bundesregierung möchte die Nachbarschaftspolitik insbesondere in Bezug auf die östlichen ENP-Staaten in diese Richtung weiterentwickeln und kann sich wie schon erwähnt die Ausweitung der Energiegemeinschaft zum Beispiel auf die Ukraine vorstellen. ${ }^{80}$ Die Energiepolitik wird ohnehin aus den oben genannten Gründen einen größeren Stellenwert in den Beziehungen zu den Nachbarn der EU einnehmen. Die ENP gilt es aber darüber hinaus als Instrument zur Förderung von Stabilität und Entwicklung hin zu mehr Demokratie und Marktwirtschaft zu nutzen und dabei gleichzeitig den wirtschaftlichen und politischen Einfluss der EU in den östlichen und kaukasischen Nachbarregionen gegenüber den Interessen Russlands aber auch denen der USA zu sichern. Der Umfang der hierbei zu bewältigenden Aufgaben lässt es allerdings eher unwahrscheinlich erscheinen, dass die Neuausrichtung der Nachbarschaftspolitik unter deutscher Ratspräsidentschaft bereits abgeschlossen werden kann, was sich auch darin zeigt, dass dieser Punkt in das längerfristige „Dreierprogramm“ mit der portugiesischen und slowenischen Präsidentschaft aufgenommen wurde..$^{81}$

Während sich die Nachbarschaftspolitik mit dem geografischen Umfeld Europas befasst, gilt es ergänzend hierzu auch Konzepte für strategisch wichtige Regionen in größerer Entfernung zu entwickeln. Die EU-Zentralasienstrategie, die, wie vom Europäischen Rat im Dezember angekündigt, bis zum Ende der deutschen Ratspräsidentschaft ausgearbeitet und verabschiedet werden soll, ${ }^{82}$ ist hierzu ein wichtiger Beitrag - zumal der diesbezügliche geografische Raum nicht nur im Hinblick auf die Krise in Afghanistan, sondern auch wegen der strategischen Versorgung der Europäischen Union mit Gas und Öl von großer Bedeutung ist.

78 Der aktuelle Stand der jeweiligen bilateralen Verhandlungen ist abrufbar unter: http://ec.europa.eu/enlargement/ (letzter Zugriff: 26.01.2007).

79 Vgl. hierzu Europäische Kommission: Mitteilungen der Kommission an den Rat und das Europäische Parlament. Über die Stärkung der Europäischen Nachbarschaftspolitik, KOM(2006)726 endgültig, S. 5-12.

80 Vgl. Glos: Energiepolitik, S. 50-53; Steinmeier: Gemeinsam die Energieversorgung sichern, in: Handelsblatt, 23.03.2006.

81 Vgl. Rat der Europäischen Union: Achtzehnmonatsprogramm, S. 63.

82 Vgl. Europäischer Rat: Schlussfolgerungen des Vorsitzes, 14./15. Dezember 2006, S. 18. 


\section{Europäische Außen- und Sicherheitspolitik}

Im Bereich der Gemeinsamen Außen-, Sicherheits- und Verteidigungspolitik bestehen die Herausforderungen und Chancen für die deutsche Ratspräsidentschaft in Lösungsversuchen ganz konkreter außenpolitischer Aufgaben und weniger in der Weiterentwicklung des bestehenden institutionellen Rahmens. Allerdings kommt es bei der Klärung zum weiteren Vorgehen in der Verfassungsfrage auf die Verankerung des Amts eines Europäischen AuBenministers und des Europäischen Diplomatischen Dienstes an, um die Gemeinsame Außen- und Sicherheitspolitik (GASP) substanziell zu stärken. Möglicherweise werden auch von anderen Mitgliedstaaten Diskussionen um Vorwegnahmen bestimmter Elemente des Verfassungsvertrages gerade im intergouvernementalen Bereich der Außenpolitik gewünscht. Die deutsche Ratspräsidentschaft wird hierauf kaum eingehen, plant aber dem Hohen Repräsentanten für die GASP, Javier Solana, zum Beispiel bei Drittlandstreffen die Vorsitzfunktion $\mathrm{zu}$ übertragen. Weitere Initiativen der Ratspräsidentschaft könnten sich befassen mit Fragen der zivil-militärischen Koordination, der Stärkung der Krisenbewältigungsstrukturen, insbesondere der EU-Planungs- und Führungsinstrumente für Missionen im Rahmen der Europäischen Sicherheits- und Verteidigungspolitik (ESVP) (Hampton Court follow-up), mit der Verbesserung der interinstitutionellen Zusammenarbeit zwischen der EU und der NATO sowie vor allem zwischen der EU und den Vereinten Nationen. ${ }^{83}$

Was die konkreten politischen Herausforderungen angeht, so könnte sich die deutsche Präsidentschaft sehr bald mit den Verhandlungen über ein neues Partnerschafts- und Kooperationsabkommen (PKA) mit Russland zu befassen haben, wo zurzeit allerdings noch die Erteilung des Verhandlungsmandats von der polnischen Regierung wegen russischer Importbeschränkungen für polnische Fleischerzeugnisse blockiert wird. Im Rahmen der Neuaushandlung des PKA wäre zu klären, ob Russland nicht doch zu einer Ratifizierung des Energiecharta-Vertrags und seines Transitprotokolls bewegt werden kann beziehungsweise ob die für die Europäische Union wichtigen Punkte der Energiecharta in das PKA aufgenommen werden können. Außerdem geht es um die Umsetzung des Konzepts der Vier Gemeinsamen Räume für Wirtschaft, innere Sicherheit, äußere Sicherheit sowie Forschung, Bildung und Kultur. Hierfür war 2005 eine Roadmap vereinbart worden, die äußerst weitreichende Ziele wie die Schaffung eines offenen und integrierten Marktes zwischen der EU und Russland und ein visafreies Reiseregime verfolgt, aber auch sehr konkrete Maßnahmen beinhaltet unter anderem in den Bereichen Marktregulierung, nukleare Sicherheit, Visaerleichterung und Rückführungsabkommen oder der Zusammenarbeit zwischen FRONTEX und der russischen Grenzpolizei.

Für die EU ist die Zusammenarbeit mit dem Nachbarn und ,strategischen Partner' Russland (und damit auch die Aushandlung eines neuen PKA) bedeutsam im Hinblick auf den Bezug von Rohstoffen, die Entwicklung des russischen Marktes für Handel und Investitionen, sowie die Zusammenarbeit mit Russland bei Fragen der internationalen Sicherheit im Rahmen der UN oder der OSZE in Bezug auf den Balkan (Kosovo-Kontaktgruppe), den postsowjetischen Raum, aber auch den Nahen Osten (Quartett) und den Mittleren Osten. Aus russischer Sicht besteht großes Interesse an der Zusammenarbeit mit der EU, um die eigene Wirtschaft zu modernisieren und um die enormen Finanzmittel für die Sicherheit und Effizienz der Produktion und des Transports von Energie aufbringen zu können, die voll-

83 Siehe u.a. Javier Solana: Follow-up on Hampton Court discussion regarding certain CFSP aspects, S416/05; Atlantic News: Nr. 3827, 28.11.2006, S. 6; Thierry Tardi: EU-UN cooperation in peacekeeping: a promising relationship in a constrained environment, Chaillot Paper, 11.07.2005, S. 49-68. 
ständige Integration in die Weltwirtschaft (vor allem in den Rahmen der WTO) zu erreichen und als sicherheitspolitischer Akteur neben den USA in einer wichtigen Rolle anerkannt zu sein. ${ }^{84}$

Weitere Schritte werden unter der deutschen Ratspräsidentschaft im Bereich der Außenwirtschaftspolitik erwartet, wobei sich die Verbindung zur deutschen G8-Präsidentschaft als hilfreich erweisen könnte. In erster Linie soll im Rahmen des transatlantischen Dialogs auf dem geplanten Gipfeltreffen zwischen der Europäischen Union und den Vereinigten Staaten Ende April über eine Vertiefung der Wirtschaftspartnerschaft in Richtung eines transatlantischen Markts beraten werden. ${ }^{85}$ Als multilaterale Initiative wird der Wiederaufnahme der Doha-Runde eine entscheidende Bedeutung beigemessen, zudem sollen aber auch Bemühungen um Handelsvereinbarungen mit den AKP-Staaten weiterverfolgt und das Assoziationsabkommen mit dem Mercosur abgeschlossen werden ${ }^{86}$ Zudem begannen Mitte Januar 2007 Verhandlungen der Europäischen Union mit China über ein Partnerschaftsabkommen. Des weiteren sind ein Gipfeltreffen mit Kanada über die außenwirtschaftlichen Beziehungen geplant, sowie ein EU-Japan Gipfel, in dessen Mittelpunkt auch die regionale Kooperation und Integration in Nord-Ostasien sowie der Austausch über die Lage in Nordkorea und die Verstetigung des Dialogs über Zukunftsfragen stehen soll. Der Dialog über Zukunftsfragen spielt übrigens auch bei China eine bedeutende Rolle in Bezug auf die Umwelt- und Energiepolitik sowie den Normen- und Werteexport hinsichtlich EU-Produktionsstandards, Gesundheits- und Arbeitsschutzbestimmungen.

Schließlich geht es auch um die Stärkung der Entwicklungszusammenarbeit zur Verbesserung der Lebensbedingungen in Afrika und anderen Weltregionen und damit um die Verminderung des Migrationsdrucks. Die EU ist hierbei aus Sicht der Bundesregierung dazu aufgerufen, einen Beitrag zur nachhaltigen Entwicklung, Armutsreduzierung und zur Erreichung der Millenniums-Entwicklungsziele zu leisten. Hierzu wird vor allem die Erhöhung der offiziellen Entwicklungshilfe und der qualitativen Verpflichtungen anzustreben sein. Auch gilt es im Rahmen der EU-Afrikastrategie Initiativen in den Bereichen Energie, Governance und für das Thema HIV/AIDS zu ergreifen. ${ }^{87}$

Die EU wird sich in den nächsten sechs Monaten zudem mit der Frage zu befassen haben, welche Rolle sie in der demokratischen Republik Kongo in Zukunft spielen kann, nachdem die Mission EUFOR RD Congo ${ }^{88}$ Ende letzten Jahres erfolgreich abgeschlossen wurde. Zumindest sollten die Missionen „EUPOL Kinshasa“ (die den Aufbau einer integrierten kongolesischen Polizeieinheit unterstützt) und EUSEC-R.D. Congo zur Sicherheitsstrukturreform, welche bis 30. Juni 2007 verlängert worden sind ${ }^{89}$ mittelfristig fortgesetzt und in eine integrierte Mission mit Polizei- und Justizelementen weiterentwickelt werden. Bei den europäisch geführten ESVP-Missionen auf dem Balkan stellt sich die Frage der weiteren Ausrichtung beziehungsweise der Entlastung europäischer Kapazitäten. Hierbei wird die Bundesregierung vor allem einen Akzent auf die schrittweise Reduktion der europäischen

84 Vgl. Heinz Timmermann: Strategische Partnerschaft: Wie kann die EU Russland stärker einbinden? in: integration 4/2002, S. 297-310, hier: S. 297.

85 Vgl. Rede der Bundeskanzlerin Angela Merkel am 17.01.2007 im Europäischen Parlament, abrufbar unter: http://www.europarl.de (letzter Zugriff: 26.1.2007).

86 Vgl. Bundesregierung: Europa gelingt gemeinsam, S. 24.

87 Ebenda.

88 Zu Hintergründen und Umfang der Mission vgl.: Daniel Göler: Europas Interessen in Zentralafrika. Die Mission „EUFOR RD Congo“, in: Dokumente 3/2006, S. 22-27.

89 Rat der Europäischen Union: Council extends the mandate of the EU police mission in Kinshasa, 08.12.2006, abrufbar unter: http://www.consilium.europa.eu/ueDocs/cms_Data/docs/pressData/en/esdp/92068.pdf (letzter Zugriff: 26.01.2007). 
Militärmission in Bosnien (EUFOR-ALTHEA) setzen. ${ }^{90}$ Andererseits gilt es, nach der Regulierung der Kosovo-Statutsfrage die Übernahme der UN-Mission im Kosovo (UNMIK) weiter vorzubereiten, die neben der militärischen ESVP-Mission ALTHEA in Bosnien-Herzegowina die größte ESVP-Herausforderung für die EU im Bereich des Verwaltungs-, Justiz- und Polizeiaufbaus darstellt und eine gute Kooperation mit der NATO erfordert. Letzteres gilt auch für die geplante zivile ESVP-Mission in Afghanistan, bei der nach Vorstellungen der Bundesregierung circa 160 Polizeioffiziere zum Einsatz kommen sollen. ${ }^{91}$

Ein weiterer Schwerpunkt der GASP wird der Nahostkonflikt sein, und zwar nicht nur wegen des überwiegend von den Europäern unter dem UN-Dach getragenen Libanon-Einsatzes (UNIFIL). Nach den jüngsten sehr positiv verlaufenen Konsultationen zwischen der Bundesregierung und den USA in Washington und in Berlin stehen für die deutsche Ratspräsidentschaft die Chancen gut, ${ }^{92}$ das Nahost-Quartett (UN, USA, EU, Russland) zu mobilisieren und die Umsetzung der Roadmap anzugehen, um den Konflikt zwischen Israel und den Palästinensern mit einem neuen Anlauf einer schrittweisen Lösung näher zu bringen.

Insgesamt sieht es nach einer Verbesserung der transatlantischen Kooperationsmöglichkeiten aus - nicht zuletzt in der Folge des Ausgangs der jüngsten Kongresswahlen und der Schwierigkeiten der USA im Irak. So wird anvisiert, sich beim EU-USA-Gipfeltreffen den Fragen des (zivilen) Krisenmanagements und Aspekten der Weltordnung (Iran, Irak, Afghanistan, Nordkorea) zu widmen, und die USA scheinen sogar das Interesse signalisiert zu haben, sich bei der künftigen EU-Kosovo-Mission zum Beispiel mit einem Polizeikontingent zu beteiligen. Auch gibt es auf amerikanischer Seite die Bereitschaft, sich gemeinsam mit der EU für eine Wiederaufnahme der Doha-Welthandelsrunde einzusetzen, die nicht zuletzt an beiden Partnern als Reaktion auf die Forderungen der Entwicklungsländer gescheitert war. Die USA scheinen unter der auslaufenden Bush-Regierung die Zusammenarbeit mit der EU als Partner zur Teilung von Lasten und Verantwortung zu suchen, was unter der deutschen Ratspräsidentschaft auch zur weiteren Entspannung zwischen den ,Atlantikern“ und ,Europäern“ in der EU führen könnte. Inwieweit Deutschland und die EU aber überhaupt zu zusätzlichen Lastenübernahmen in der Lage sind, sollte mit Vorsicht bedacht werden.

\section{Die europäische Verfassungsfrage}

Den Verfassungsprozess zu einem erfolgreichen Ende zu führen ist seit Amtsantritt der großen Koalition ein zentrales Anliegen der Bundesregierung. Neben der Überzeugung, dass der vorliegende Vertrag das Optimum des inhaltlich Machbaren darstellt ${ }^{93}$, dürften beim Festhalten am Verfassungsvertrag auch taktische Erwägungen eine Rolle gespielt haben, um den Druck auf die ,Problemländer` zu erhöhen. Allerdings haben sich die Hoffnungen auf eine einfache Wiedervorlage beziehungsweise ein zweites Referendum vor allem in Frankreich als unrealistisch erwiesen. Die sozialistische Präsidentschaftskandidatin Royal hat zum Beispiel in Äußerungen immer wieder betont, dass sie einer unveränderten Neuauflage des Verfassungsprojektes nicht zustimmen würde. ${ }^{94}$ Sie könnte sich aber einen Reform-Vertrag

90 Vgl. Atlantic News: Nr. 3838, 18.01.2007, S. 3.

91 Vgl. Atlantic News: Nr. 3837, 16.01.2007, S. 3; Frankfurter Allgemeine Zeitung: Afghanistan: EU erwägt Polizeimission, 22.01.2007.

92 Vgl. Christian Wernicke: Merkel-Besuch in Washington: Atlantisches Quartett-Spiel, in: Süddeutsche Zeitung, 05.01.2007; Der Tagesspiegel: Rice in Berlin. Merkel trifft US-Außenministerin, 18.01.2007.

93 Vgl. Regierungserklärung von Angela Merkel vor dem Deutschen Bundestag vom 30. November 2005, Bundestags-Plenarprotokoll, 16. Wahlperiode, 4. Sitzung, S. 88. 
mit einer sozialpolitischen Erklärung vorstellen, welche die Rechte der Arbeitnehmer berücksichtigt, der aus ihrer Sicht dann den unsicheren Weg eines zweiten Referendums nehmen müsste. ${ }^{95}$ Der gaullistische Kandidat Sarkozy hat durch seine verschiedenen Initiativen zu einem Mini-Traité ${ }^{6}$ deutlich gemacht, dass Frankreich unter seiner Führung zwar aktiv an der Lösung der Verfassungskrise mitarbeiten würde, dass er aber eine Alternative zum gegenwärtig umfänglichen Verfassungstext bevorzugt. Auch in den Niederlanden gibt es keinerlei Anzeichen für eine Wiedervorlage der Verfassung, zumal entsprechende Meinungsumfragen immer noch eine deutliche Ablehnung zeigen. ${ }^{97}$ Da zudem die Länder, in denen die Ratifizierung oder gar ein Referendum noch ansteht, wie Irland, Dänemark und Portugal ${ }^{98}$, wenig oder gar keine Bemühungen zur Fortsetzung beziehungsweise Aufnahme des Ratifizierungsprozesses zeigen, solange keine Lösung in Frankreich und den Niederlanden in Sicht ist, zeichnet sich für die deutsche Ratspräsidentschaft eine Situation ab, in der nach Modellen zur Bewahrung der Substanz des Verfassungsvertrages gesucht werden muss.

Die wichtigste Herausforderung wird sein, in den der deutschen Präsidentschaft vom Europäischen Rat aufgetragenen Konsultationen mit allen Mitgliedstaaten die Konsenslinien herauszuarbeiten. ${ }^{99}$ Ein nicht zu unterschätzendes Problem besteht darin, dass die Bundesregierung natürlich auch die Staaten berücksichtigen muss, die der Verfassung bereits zugestimmt haben, deren positive Haltung zu einem reduzierten Vertrag aber wieder in Frage gestellt werden könnte. Wichtige in diesem Zusammenhang zu erörternde Fragen werden unter anderem sein, ob der Name des Verfassungsvertrags zu ändern ist - was auch von Bundesaußenminister Steinmeier schon einmal angedacht wurde, ${ }^{100}$ oder wie Bedenken einzelner Staaten durch spezielle Erklärungen etwa zum Schutz der nationalen Identitäten entgegengekommen werden kann.

Ferner wäre zu erörtern, ob eine Beschränkung auf den ersten Teil des Verfassungsvertrags sinnvoll ist und inwieweit dabei mit Blick auf Vorbehalte in der britischen Bevölkerung der Grundrechtecharta durch bloß einen entsprechend zu formulierenden Verfassungsartikel Primärrechtsstatur zugewiesen werden könnte. ${ }^{101}$ Des Weiteren gilt es zu bedenken, wie ein solcher Vertrag gestaltet sein müsste, um die Zahl einzelstaatlicher Referenden auf ein Mindestmaß zu reduzieren.

Nach Abschluss solcher Konsultationen könnte dann in einem zweiten Schritt ausgelotet werden, welche Gesamtpakete sich aus den einzelnen Positionen schnüren ließen. Die Bundesregierung sollte dabei trotz aller Diskussionen um einen Mini-Traité und die vielfach angemahnte Konzentration auf die institutionellen Reformen nicht aus dem Auge verlieren, dass gerade die Einbeziehung weiterer Politik-Materien die Problemlösung erleichtern

94 Vgl. Jean Quatremer: Pour Ségolène Royal, l'Europe ne manque pas de Constitution, in: Liberation, 13.09.2006.

95 Vgl. L'Express: Ségolène Royal veut un nouveau référendum sur l'Europe en 2009, 19.01.2007.

96 Rede von Nicolas Sarkozy vor den Amis de l'Europe und der Fondation Schumann, 08.09.2006, abrufbar unter: http://www.robert-schuman.org/actualite/bruxelles/discours8sept.pdf (letzter Zugriff: 25.11. 2006).

97 Vgl. Mark Beunderman: Dutch Open to New-Style EU Constitution, Poll Shows, in: euobserver.com, 19.01.2007.

98 In Polen und Tschechien ist die Form der Ratifizierung noch nicht abschließend entschieden; in Großbritannien hat sich Tony Blair zwar auf ein Referendum festgelegt, ob sein Nachfolger sich diesbezüglich gebunden fühlt, ist aber noch offen.

99 Vgl. Europäischer Rat: Schlussfolgerungen des Vorsitzes, 15./16. Juni 2006, S. 17.

100 Vgl. EurActiv.com: Verfassung: Außenminister wollen Einigung bis 2009, 29.05.2006.

101 So ein Vorschlag des deutschen Europaabgeordneten und ehemaligen Konventsmitglieds Elmar Brok. Vgl. Frankfurter Allgemeine Zeitung: Berlin will Rasche Entscheidung über die EU-Verfassung, 03.01.2007. 
könnte. ${ }^{102}$ Konkret würde es in der aktuellen Situation zum Beispiel darauf ankommen, ob polnische Widerstände gegen die doppelte Mehrheit ${ }^{103}$ durch ein Entgegenkommen beim Aufbau einer gemeinsamen Energiepolitik mit entsprechender vertraglicher Verankerung aufgelöst werden könnten. Ebenso könnten Bedenken in der französischen Bevölkerung durch ein Sozialprotokoll oder aber besondere sozialpolitische Opt-In-Regelungen entkräftet werden - die allerdings so auszugestalten wären, dass sie nicht neue Widerstände in Großbritannien hervorrufen.

Der Schwerpunkt der deutschen Ratspräsidentschaft wird ausgehend vom Verfassungsvertrag auf der informellen Auslotung ${ }^{104}$ solcher Kompromisse liegen. Die dadurch gewonnenen Erkenntnisse ließen sich dann zum Ende der deutschen Präsidentschaft zu einem Gesamtpaket zusammenfassen, das Grundlage für eine möglicherweise sehr zügige Einigung sein könnte. ${ }^{105}$ Eine rasche Einigung würde den ursprünglichen vom Europäischen Rat im Juni 2006 vereinbarten Zeitplan etwas beschleunigen, ${ }^{106}$ der eine Lösung der Verfassungskrise spätestens für die zweite Jahreshälfte 2008 unter französischer Ratspräsidentschaft anvisierte, und hätte den entscheidenden Vorteil, dass die neuen Regelungen nach einer circa anderthalbjährigen Ratifizierungsphase bereits ab 2009 wirksam werden könnten, was nach wie vor das erklärte Ziel der Bundesregierung ist. ${ }^{107}$

Sollte sich die oben angedachte Beschleunigung des Zeitplans als nicht machbar erweisen, weil es zusätzlichen Beratungsbedarf gibt, so wäre die deutsche Ratspräsidentschaft angehalten, eine konkrete ,Roadmap“ für die weiteren Verhandlungen auszuarbeiten. Neben der Erhaltung der inhaltlichen Substanz des Verfassungsvertrages sollte die Bundesregierung dabei auch das Ziel der Vereinfachung der komplexen Primärrechtsstruktur aus Gründen der Verständlichkeit für den Bürger verfolgen. ${ }^{108}$ Denn jede Reform, die nicht die bestehenden Verträge komplett ersetzt, würde das Nebeneinander verschiedener Verträge sowie die Vermischung von grundlegenden Normen und Ausführungsbestimmungen im Primärrecht nicht auflösen. Aus diesem Grund könnte ein mehr- oder zumindest zweistufiges Verfahren vorgeschlagen werden, nachdem zunächst - bis zu den Europa-Wahlen 2009 - die inhaltlichen und institutionellen Verfassungsreformen umgesetzt werden.

Ob dies dann am Besten je nach zu wählender Alternative in einer vereinfachten Verfassung im Sinne des ersten Teils des durch die Regierungskonferenz 2003/04 überarbeiteten Konventsentwurfes, eines neuen Kopfvertrages, eines Mantelvertrages oder aber einer Integration der substanziellen Reformen des Verfassungsprojekts in die bestehenden Verträge erfolgen sollte, bliebe noch hinsichtlich der jeweiligen Auswirkungen auf Bestandserhaltung oder Veränderungsbedarf des existierenden Primärrechts zu prüfen. ${ }^{109}$ Dabei sollte man sich

102 Vgl. Daniel Göler/Mathias Jopp: Die europäische Verfassungskrise und die Strategie des ,langen Atems‘, in: integration 2/2006, S. 91-105, hier: S. 99.

103 Vgl. Helmut P. Gaisbauer: Der EU Konvent aus der Perspektive beitrittswilliger Staaten - Schlüsselinstitution einer Westerweiterung, in: Forum Parlament 1/2005, S. 10-14, hier: S. 14.

104 Vgl. Mitschrift der Pressekonferenz von Bundeskanzlerin Merkel und Ministerpräsident Balkenende vom 28.10.06, abrufbar unter: www.bundesregierung.de (letzter Zugriff: 10.01.2007).

105 Vgl. die Äußerungen des deutschen Ständigen Vertreters bei der EU, Wilhelm Schönfelder, und die ähnliche Auffassung des CDU-Europaabgeordneten Elmar Brok, in: Berlin will rasche Entscheidung über die EU-Verfassung, in: Frankfurter Allgemeine Zeitung, 03.01.2007.

106 Vgl. Europäischer Rat: Schlussfolgerungen des Vorsitzes, 15./16. Juni 2006, S. 17.

107 Vgl. Frankfurter Allgemeine Zeitung: Deutsche Ratspräsidentschaft. Merkel: EU Verfassung bis 2009, 11.10.2006.

108 Zur Debatte um eine Vereinfachung der Verträge vgl.: Daniel Göler: Die neue europäische Verfassungsdebatte. Entwicklungsstand und Optionen für den Konvent, Bonn 2002, S. 51-56.

$109 \mathrm{Zu}$ den verschiedenen Optionen vgl.: Daniel Göler/Mathias Jopp: Die europäische Verfassungskrise, S. 91105. 
gleichzeitig darauf einigen, einen nächsten Schritt einzuplanen mit dem Ziel einer verbindlichen Strukturrevision beziehungsweise einer Überarbeitung und Vereinfachung der reformierten Verträge. So könnte am Ende dann ein dem jetzigen Verfassungsvertrag sehr ähnliches Dokument stehen, womit das Ziel der Bundesregierung, den Verfassungsprozess zu einem erfolgreichen Ende zu führen, zumindest mittelfristig erreicht würde.

\section{Ziele und Inhalt der Berliner Erklärung}

Neben dem Frühjahrs- und Juni-Gipfel wird es während der deutschen Ratspräsidentschaft auch eine Sitzung der Staats- und Regierungschefs anlässlich des 50. Jahrestages der Unterzeichnung der Römischen Verträge in Berlin geben. Auf diesem Sondergipfel wird gemäß den Schlussfolgerungen des Europäischen Rates vom Juni 2006 die Berliner Erklärung verabschiedet. ${ }^{110}$ Ziel dieser Erklärung soll es sein, vor dem Hintergrund der letzten fünfzig Jahre eine Bilanz zu ziehen und den Mehrwert des europäischen Integrationsprojekts zu verdeutlichen, um so einen Beitrag zur Überwindung der aktuellen Akzeptanzkrise zu leisten. Darüber hinaus wird die Erklärung aber auch die Gelegenheit bieten über die Grundzüge der weiteren Integration zu reflektieren, so dass die Bundesregierung hierin eine Gelegenheit zur „Orientierung über Werte und Aufgaben der EU“ sieht, ${ }^{111}$ welche durch eine „Verständigung über historische und kulturelle Grundlagen der europäischen Idee und über europäische Interessen [...] das Zusammengehörigkeitsgefühl Europas festigen“"112 soll.

Demnach wären zunächst die wesentlichen Leistungen des bisherigen Integrationsprozesses herauszustellen: Die Sicherung von Frieden und Stabilität in Europa, die Förderung von Prosperität und Wohlstand in den europäischen Volkswirtschaften, die Schaffung eines Rechts- und Freiheitsraums sowie die Entwicklung einer gesamteuropäischen Wertegemeinschaft. Viele dieser Punkte mögen auf den ersten Blick zwar rückwärtsgewandt klingen, sind aber gerade für die Stabilität des neuen vereinten Europa von besonderer Bedeutung. Zweitens sollten die Herausforderungen der Globalisierung einen wesentlichen Stellenwert in der Berliner Erklärung erhalten, denn jene erweisen sich als neue zusätzliche Begründung des europäischen Einigungsprojektes, neben der immer noch sehr bedeutsamen Friedenssicherungsfunktion zwischen den europäischen Staaten. Die neuen Herausforderungen durch Klimawandel und Ressourcen- beziehungsweise Energieverknappung, globale Umweltverschmutzung, internationalen Terrorismus, transnationale Kriminalität, Proliferation von Massenvernichtungswaffen sowie regionale Konflikte und Kriege sind von keinem der europäischen Staaten allein zu meistern, auch nicht von den größten unter ihnen. Gleiches gilt für die Frage der Organisation der globalen Wirtschafts- und Handelsordnung. Wenn die europäischen Staaten hier Gestaltungswillen sowie auch Gestaltungsmacht an den Tag legen wollen, werden sie dies nur gemeinsam durch die EU erreichen, wie es ja auch schon im Motto des deutschen Präsidentschaftsprogramms zum Ausdruck gebracht wird. Pointiert könnte eine solche neue oder zusätzliche Begründung der europäischen Integration in der Formel ,Europäisierung als Antwort auf die Globalisierung' zusammengefasst werden. Dabei geht es nicht um einen romantisierenden europäischen Sozialprotektionismus oder den Ausbau der Europäischen Union zu einer Antiglobalisierungsfestung, sondern um die aktive Mitgestaltung globaler Prozesse. Denn ebenso unaufhaltsam wie die Globalisierung sich

110 Vgl. Europäischer Rat: Schlussfolgerungen des Vorsitzes, 15./16. Juni 2006, S. 10.

111 Erklärung des Bundeskabinetts zur deutschen EU-Ratspräsidentschaft, Pressemitteilung Nr. 387 des Presseund Informationsamtes der Bundesregierung, abrufbar unter: www.bundesregierung.de (letzter Zugriff: 26.01.2007).

112 Ebenda. 
vollzieht, so offensichtlich ist auch die Tatsache, dass sie einer politischen, wirtschaftlichen (und sozialen) Lenkung bedarf, wobei die Europäische Union eine führende Rolle einnehmen kann.

\section{Schlussbetrachtung}

Angesichts der Fülle der Problemfelder und der immer miteinzubeziehenden Möglichkeit unerwarteter Krisen und Ereignisse, welche Aufmerksamkeit und Verhandlungsressourcen binden, wird die deutsche Ratspräsidentschaft wohl kaum alle Bereiche ihres Programms abarbeiten können. Hier zeigt sich auch die „Weisheit“ des Projekts eines detaillierten 18Monatsprogramms mit den beiden nachfolgenden Präsidentschaften. ${ }^{113}$

Es ist ohnehin schwierig alle Erwartungen zu erfüllen. So wurde von deutscher Gewerkschaftsseite schon frühzeitig angemahnt, dass die Bundesregierung in ihrer Präsidentschaft mehr sozialpolitische Ambitionen an den Tag legen ${ }^{114}$ und einen Schwerpunkt im Bereich der sozialen Dimension setzen solle. Von Seiten der Umweltverbände wird immer wieder beklagt, dass die Bundesregierung im Umwelt- und Klimaschutz weitaus ambitionierte Initiativen ergreifen müsste. Betrachtet man die deutsche Haltung bezüglich der nationalen Allokationspläne für Treibhausgasemissionen im Zeitraum 2008-2012 wird sogar die Befürchtung vertreten, die Bundesregierung könnte sich zum klimapolitischen Bremser entwickeln. ${ }^{115}$ Industrie und Energieproduzenten kritisieren hingegen zu hohe Umwelt- und Klimaschutzauflagen und sehen sie als Kostenbelastung und Wettbewerbsnachteile. Andererseits hat die Wirtschaft aber auch hohe Erwartungen hinsichtlich erfolgreicher Abschlüsse der Handelsvereinbarungen mit den USA, China und Russland sowie hinsichtlich der Wiederaufnahme der Doha-Runde, wobei freilich schon allein aufgrund der teilweise schwierigen Partner offen ist, inwieweit die handelspolitische Offensive Europas bereits unter deutscher Ratspräsidentschaft zum Erfolg führt.

Wenn eine Präsidentschaft alle an sie gerichteten Ansprüche und Wünsche verwirklichen könnte, wäre dies ein Wunder. Es gilt deshalb, sich ein Stück weit von (auch berechtigter) Kritik oder bestimmten Verbandsinteressen zu lösen und den Blick auf die großen Zielperspektiven zu konzentrieren. Gerade in der Fähigkeit dies zu tun lag in der Vergangenheit eine der Haupttugenden deutscher Europapolitik und erfolgreicher EU-Präsidentschaften. ${ }^{116}$ Die Möglichkeiten, dass der Bundesregierung dies erneut gelingt, können als durchaus positiv eingeschätzt werden. Denn es gibt in allen Bundesministerien einen erheblichen Ehrgeiz nach eineinhalb bis zweijähriger Vorbereitungszeit, in der deutschen Präsidentschaft substanzielle Ergebnisse zu erzielen. Erleichtert werden könnte dies durch die Erwartung vieler Partner in der Europäischen Union, dass die deutsche Regierung zur Zeit das einzige Aktionszentrum ist, das Europa aus der Krise führen kann und will. Gerade dies eröffnet die Chance, die Akzeptanz deutscher Initiativen bei den Mitgliedstaaten zu erhöhen. Hinzu

113 Martin Winter: EU-Ratspräsidentschaft. Viel Balsam für des Bürgers Seele, in: Süddeutsche Zeitung, 22.12. 2006.

114 Vgl. hierzu die gemeinsame Erklärung vom DGB-Vorsitzenden Michael Sommer und Ministerpräsident Kurt Beck (SPD) zu den Präsidentschaften Deutschlands in EU und G8 vom 15.11.2006, abrufbar unter: http:// www.dgb.de/presse/pressemeldungen/pmdb/pressemeldung_single?pmid=2868 (letzter Zugriff: 26.01.2007).

115 Vgl. Offener Brief an führende Umweltverbände gegen die Position der Bundesregierung zum nationalen Allokationsplan an Kurt Beck, abrufbar unter: www.nabu.de (letzter Zugriff: 26.01.2007).

116 Vgl. Daniel Göler: Europapolitik im Wandel. Deutsche Integrationsmotive und Integrationsziele nach der Wiedervereinigung, Münster 2004, S. 63; Heinrich Schneider/Mathias Jopp/Uwe Schmalz (Hrsg.): Eine neue deutsche Europapolitik. Rahmenbedingungen - Problemfelder - Optionen, Bonn 2001; Mathias Jopp/Uwe Schmalz: Deutsche Europapolitik 2000. Prioritäten - Positionen - Perspektiven, in: Aus Politik und Zeitgeschichte 6/2000, S. 12-19. 
kommt, dass gegenwärtig eine ganze Reihe von Rahmenbedingungen sehr günstig sind: Der Wirtschaftsaufschwung im Euro-Raum eröffnet größere Handlungsspielräume, die Erweiterung ist mit dem Beitritt Rumäniens und Bulgariens zu einem vorläufigen Ruhepunkt gekommen (mit Ausnahme eventuell Kroatiens), im Nahostkonflikt könnte die EU mit Unterstützung der USA gegebenenfalls Fortschritte erzielen, und die veränderten politischen Verhältnisse in den USA lassen ohnehin eine etwas konstruktivere transatlantische Zusammenarbeit in weltpolitischen Fragen erwarten. Hinzu kommt, dass aufgrund aktueller Anlässe auch unter den EU-Mitgliedstaaten eine größere Bereitschaft zu einem Vorangehen in einzelnen Politikbereichen, wie der Energiepolitik oder der Innen- und Justizpolitik zu erkennen ist. Wenn diese günstigen Rahmenbedingungen zu substanziellen Fortschritten in den oben erörterten Politikbereichen führen und es zudem noch gelingen sollte, den Verfassungsprozess wieder auf die Spur zu setzen, könnte die deutsche Ratspräsidentschaft ein echter Erfolg werden.

Dieser Beitrag entstand im Rahmen des Forschungsprojekts „Ein Europa der Bürger Verfassung und effiziente Politik", das vom Institut für Europäische Politik (IEP) und der ASKO EUROPA-STIFTUNG gemeinsam durchgeführt wird sowie des Forschungsprojekts „Europapolitische Leitbilder in der erweiterten Europäischen Union”, das vom IEP, dem Jean Monnet Lehrstuhl für Politische Wissenschaft und Europäische Fragen an der Universität zu Köln sowie der Karls Universität Prag durchgeführt und von der VolkswagenStiftung gefördert wird.

\title{
Verschiebung der Kräfteverhältnisse
}

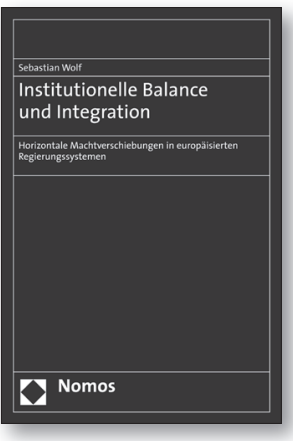

\author{
Institutionelle Balance und Integration
}

Horizontale Machtverschiebungen

in europäisierten Regierungssystemen

Von Sebastian Wolf

2006, 150 S., brosch., 29,- €, ISBN 978-3-8329-2161-3

Das Buch analysiert die Verschiebung der Kräfteverhältnisse zwischen den an der Gesetzgebung beteiligten Verfassungsorganen der EU-Mitgliedstaaten im Prozess der europäischen Integration. Im Zentrum des Werkes stehen 15 vergleichende Länderstudien.

Bitte bestellen Sie bei Ihrer Buchhandlung oder bei Nomos 